\title{
Leerstelle Kochbuch. Der Mangel an Kulinaria in öffentlichen Bibliotheken
}

„Es wurde leider keine Bibliothek gefunden, die den gesuchten Titel besitzt“, meldet WorldCat für die zweite überarbeitete Ausgabe des Dieter Müller Kochbuch. Feine Küche leichtgemacht. 180 Rezepte aus den Schweizer Stuben in Wertheim aus dem Jahr 1988. ${ }^{1}$ Die erste Ausgabe aus dem Jahr 1984 verzeichnet die weltgrößte bibliografische Datenbank überhaupt nicht. Das Standardwerk von Dieter Müller, einem der ersten deutschen Köche, der drei Michelin-Sterne erhielt, fehlt in vielen öffentlichen Bibliotheken, obwohl sein Kochbuch von 1984 nicht zu den Rara gehört. Das Werk steht laut WorldCat überhaupt nur in fünf deutschen Bibliotheken zur Nutzung bereit, außer in der Deutsche Nationalbibliothek (Frankfurt am Main/Leipzig) nur in Fulda und München. ${ }^{2}$ Die Auflage von 2005 besitzt keine öffentliche Bibliothek in Deutschland, sondern nur die Liechtensteinische Bibliothek.

Ähnliches gilt für alle Protagonisten des so genannten Deutschen Küchenwunders der 1970er Jahre. ${ }^{3}$ Eckart Witzigmanns Tantris-Kochbuch aus dem Jahr 1978 besitzen nur die Nationalbibliothek als zentrale Archivbibliothek Deutschlands sowie in München die Bayerische Staatsbibliothek und die dortige Universitätsbibliothek der Technischen Universität sowie die Universitätsbibliothek von Frankfurt am Main. ${ }^{4}$ Für Harald Wohlfahrts Buch Feines aus meiner Küche ist die Lage kaum anders. Außer in der Deutschen Nationalbibliothek ist der

1 Vgl. www.worldcat.org (13.10.2017). Zu Dieter Müller vgl. Zipprick, Jörg: Die Erfinder des guten Geschmacks. Eine Kulturgeschichte der Köche. Köln: Eichborn 2013, S. 259-260; Seitz, Erwin: Die Verfeinerung der Deutschen. Eine andere Kulturgeschichte. Berlin: Insel 2011, S. 726; vgl. auch autobiografisch: Müller, Dieter: Wie Deutschland genießen lernte ... Köln: Lingen 2014.

2 WorldCat nennt die Hochschul- und Landesbibliothek Fulda, die Stadtbibliothek Hannover, die Bayerische Staatsbibliothek, die Universitätsbibliothek der Technischen Universität München und die Deutsche Nationalbibliothek, Leipzig/Frankfurt am Main. Die Onlinerecherche auf der Webseite der Stadtbibliothek Hannover erbrachte kein Resultat, sodass dort das Buch vermutlich nicht mehr im Bestand vorhanden ist.

$3 \mathrm{Zu}$ den ersten Akteuren des deutschen Küchenwunders zählt Müller, Wie Deutschland genießen lernte ... (wie Anm. 1), S. 22-23: Eckart Witzigmann im Tantris (München), Henry Levy im Maître (Berlin), Jean-Claude Bourgueil in den Walliser Stuben (Düsseldorf) und seinen Bruder Jörg Müller sowie sich selbst in den Schweizer Stuben (Wertheim).

4 Selbst die Taschenbuchausgabe (Eckart Witzigmann: Meisterwerke aus der Drei-Sterne-Küche. Meine Tantris-Rezepte. München: Heyne 1986) findet sich nur in der Deutschen National-

ว Open Access. () 2018 Josef Matzerath, publiziert von De Gruyter. (c) BY-NC-ND Dieses Werk ist lizenziert unter der Creative Commons Attribution-NonCommercial-NoDerivatives 4.0 Lizenz. 
Band nur in München in der Bayerischen Staatsbibliothek und der Bibliothek der dortigen Technischen Universität vorhanden. Zu Henry Levys Maitre-Kochbuch aus dem Jahr 1980 meldet WorldCat: „Keine Informationen über Bibliotheken gefunden, die diesen Titel besitzen.“ Nur die Ausgabe von 1985 ist in der Deutschen Nationalbibliothek sowie in der Bibliothek der Technischen Universität Berlin, in München in der Bayerischen Staatsbibliothek und der Bibliothek der Ludwig-Maximilians-Universität sowie in der Universitätsbibliothek von Frankfurt am Main vorhanden. Selbst das Buch Die Philosophie der großen Küche von Jean-Claude Bourgueil, der schon 1974 für die Walliser Stuben (Düsseldorf) den zweiten Michelin-Stern erkochte, aber erst nach dem Jahr 2000 Bücher publiziert hat, weist WorldCat nur für die Staatsbibliothek Berlin, die Universitätsbibliothek Bayreuth, die Württembergische Landesbibliothek in Stuttgart und selbstverständlich für die Deutsche Nationalbibliothek aus.

Ähnlich schwierig ist die Lage für ältere Kochbücher des 20. Jahrhunderts. Alfred Walterspiels Meine Kunst in Küche und Restaurant, das Kochbuch von Deutschlands bedeutendstem Koch aus der ersten Hälfte des 20. Jahrhunderts, ${ }^{5}$ wurde 1952 gleich zweimal gedruckt und erlebte bis 1981 acht Auflagen. Laut WorldCat steht das Werk nur in acht öffentlichen Bibliotheken Deutschlands zur Verfügung. Vier dieser Bibliotheken befinden sich in München, wo Walter-

bibliothek, in München in der Bayerischen Staatsbibliothek, der Universitätsbibliothek der Ludwig-Maximilians-Universität und der Universitätsbibliothek von Frankfurt am Main.

$5 \mathrm{Zu}$ Alfred Walterspiel vgl. Siebeck, Wolfram: Die Deutschen und ihre Küche. Berlin: Rowohlt 2007, S. 117; Barlösius, Eva: Soziale und historische Aspekte der deutschen Küche. In: Mennell, Stephen: Die Kultivierung des Appetits. Die Geschichte des Essens vom Mittelalter bis heute. Frankfurt am Main: Athenäum 1988. S. 423-444, hier S. 437-438; Möckl, Karl: Die große deutsche Küche. Formen des Eßverhaltens seit den siebziger Jahren. In: Vom Hungerwinter zum Schlaraffenland. Aspekte einer Kulturgeschichte des Essens in der Bundesrepublik Deutschland. Hrsg. von Wolfgang Protzner. Wiesbaden: Franz Steiner 1987. S. 49-64, hier S. 52-55; Paczensky, Gert von u. Anna Dünnbier: Kulturgeschichte des Essens und Trinkens. München: Orbis 1994, S. 522; Heckh, Karl: Eine Fußbank für die Dame. Eine kulinarische Revue. Stuttgart: Schuler Verlagsgesellschaft 1969, S. 67; Peter, Peter: Kulturgeschichte der deutschen Küche. München: C. H. Beck 2008, S. 168-169; Walterspiel, Otto: Ein offenes Haus. Meine Kindheit im Hotel Vierjahreszeiten. Köln: Bastei Lübbe 2012, S. 26-35, 44-55 u. 165-166; Walterspiel, Karl Th.: Zwischen Ankunft und Abreise. Ein Leben für die Gastlichkeit. Norderstedt: Books on Demand 2004, S. 9-27; Zipprick, Erfinder (wie Anm. 1), S. 154; vgl. auch autobiografisch: Walterspiel, Alfred: Meine Kunst in Küche und Restaurant. München: [Selbstverlag] 1952, S. 9-13; hier S. 427: „Pfirsich Melba ist die schönste der Platten unseres alten Freundes Escoffier, die er zu Ehren der großen Melba kreierte. Nicht nur Escoffier selbst, sondern auch die Melba persönlich erzählten mir, welchen Beifall dieses in seiner Einfachheit so herrliche Gericht fand.“ Abbildungen von Werken Walterspiels aus dem Jahr 1908 finden sich bei: Friebe, Carl: Die kalte Küche. Handbuch der modernen Anrichtekunst. Nordhausen: Killinger [o. J.], S. 509: Vorspeisen „Kaiserhof“, Masthuhn „Kaiserhof“. 
spiel von 1922 bis 1960 lebte und arbeitete. Rechnet man noch die Deutsche Nationalbibliothek heraus, ${ }^{6}$ gibt es eine Ausgabe des Werkes aus dem Jahr 1952 in der Sächsischen Landesbibliothek - Staats- und Universitätsbibliothek Dresden und eine Ausgabe von 1978 in der Technischen Informationsbibliothek Hannover. ${ }^{7}$ Walterspiels Kochbuch findet sich hingegen in der British Library, der Universitätsbibliothek Bern, der Königlichen Bibliothek in Den Haag, der Universitätsbibliothek von Amsterdam und in 14 US-amerikanischen Bibliotheken. ${ }^{8}$ Die Gastronomische Bibliothek Alfred Walterspiel ist 1984 vom Auktionshaus Hartung \& Karl versteigert worden ${ }^{9}$ und ein umfangreicher handschriftlicher Nachlass steht der Forschung auch nicht zur Verfügung. ${ }^{10}$

Franz Pfordte, ${ }^{11}$ der renommierteste Gastronom des späten Kaiserreichs, wurde von zeitgenössischen Literaten vielfach gerühmt. Sein posthum 1927 erschienenes Kochbuch besitzen in Deutschland die SLUB Dresden, die Stadtbibliothek Hannover, die Universitätsbibliothek in Frankfurt am Main, die Staatsund Universitätsbibliothek Hamburg und die deutsche Nationalbibliothek. ${ }^{12}$ Ein handschriftlicher Nachlass Pfordtes ist nicht überliefert. ${ }^{13}$

6 Die Deutsche Nationalbibliothek besitzt außer der Auflage von 1967, die sich in keiner deutschen Bibliothek findet, alle anderen Ausgaben von Meine Kunst in Küche und Restaurant.

7 Vgl. www.worldcat.org (13.10.2017).

8 HCL Technical Services, Harvard College Library, Cambridge, Massachusetts, USA; University of Vermont, Bailey/Howe Library, Burlington, USA; City College/CUNY, New York, USA; Culinary Institute of America, Conrad N. Hilton Library, Hyde Park, New York, USA; Fairfield University, DiMenna-Nyselius Library, Fairfield, Connecticut, USA; New York Public Library System, NYPL, New York, USA; Library of Congress, Washington, USA; Miami University, Hamilton Campus, Rentschler Library, Hamilton, Ohio, USA; Purdue University Library, West Lafayette, Indiana, USA; University of Iowa Libraries, Iowa City, Iowa, USA; University of Missouri, Ellis Library, Columbia, Missouri, USA; University of Florida, Gainesville, Florida, USA; Texas A\&M University, Evans Library \& Annex; Main campus library complex College Station, Texas, USA; Los Angeles Public Library, Los Angeles, Kalifornien, USA.

9 Vgl. Auktionskatalog Gastronomische Bibliothek Alfred Walterspiel. Auktion 47 vom 6. November 1984. München: Auktionshaus Hartung \& Karl 1984.

10 Eine Suchanfrage auf „Kalliope-Verbund“ ergibt lediglich elf Briefe von und sechs Briefe an Alfred Walterspiel.

$11 \mathrm{Zu}$ Pfordte vgl. Maassen, Georg von: Weisheit des Essens. München: Kurt Wolff 1928, S. 233-243; Geisser, C.: Franz Pfordte. In: Pfordte, Franz: Kochrezepte. Hamburg: Verlagsbuchhandlung Broschek \& Co. 1927, ohne Seitennummerierung am Anfang des Buches; Lüth, Erich: Das Atlantic Hotel zu Hamburg 1909-1984. München: Faber 1984, S. 29-46; Walterspiel, Alfred: Meine Kunst in Küche und Restaurant. München: Südwest Verlag 1978, S. 11-12.

12 Darüber hinaus findet sich Pfordte, Kochrezepte (wie Anm. 11) in der New York Public Library, New York, USA und in der University of Utah, J. Willard Marriott Library, Salt Lake City, Utah, USA.

13 Eine Suchanfrage auf „Kalliope-Verbund“ liefert kein Resultat. 
Im Kontrast zu solchen Befunden stehen das inzwischen breite öffentliche Gespräch und der fächerübergreifende wissenschaftliche Diskurs über die Ernährung im deutschsprachigen Bereich. Spätestens seit den 1970er Jahren wurde in der Bundesrepublik Kulinarisches als Thema gesellschaftsfähig. Wie sehr es inzwischen Allgemeingut geworden ist, zeigte sich, als im Sommer 2016 Wolfram Siebeck, der prominenteste Initiator und Protagonist der deutschen Debatte über Kochkunst und Tafelkultur, verstarb. Die Feuilletons der überregionalen Zeitungen würdigten den "Gastronomiekritiker schlechthin“ (taz), ${ }^{14}$ den „Erzieher der Deutschen“ (Süddeutsche Zeitung), ${ }^{15}$ den „schreibenden Querdenker“ (Neue Zürcher Zeitung), ${ }^{16}$ den „Propheten [französischer] Köstlichkeiten“, der „Deutschland [...] kulinarisch nach Westen öffnen“ wollte (Die Zeit), ${ }^{17}$ den „schreibenden Herold der französischen Spitzenküche“ und Kämpfer „gegen Fastfood, Massentierhaltung und kulinarische Ignoranz“ $(F A Z) .{ }^{18}$ Die Tagesschau der ARD meldete am 7. Juli 2016, der Publizist und Gastronomiekritiker Wolfram Siebeck habe die Lust am Essen gesellschaftsfähig gemacht und gegen Fastfood, Fertiggerichte und Nahrungsmittelzusätze polemisiert. ${ }^{19}$

Wie etablierte Medien auf den Tod Wolfram Siebecks reagierten, belegt auch ohne Anspruch auf Vollständigkeit, dass im deutschsprachigen Raum ein öffentlicher Diskurs über kulinarische Ästhetik stattfindet. ${ }^{20}$ Über das Niveau der Kochsendungen im Fernsehen ${ }^{21}$ sowie unzähliger Hobbyküchen- und Werbewebseiten $^{22}$ hinaus finden sich seriöse Debattenbeiträge ebenso im Format

14 Kabisch, Jörn: Ein sehr ernsthafter Hedonist. http://www.taz.de/Zum-Tod-von-WolframSiebeck/!5319426/ (9.7.2016).

15 Tieschky, Claudia: Der Vorkoster. http://www.sueddeutsche.de/panorama/nachruf-der-vorkoster-1.3069134 (9.7.2016).

16 Die Neue Zürcher Zeitung druckte die DPA-Meldung unter der Überschrift Kämpfer gegen Fast Food gestorben. https://www.nzz.ch/panorama/menschen/gastrokritiker-wolfram-siebeck-kaempfer-gegen-fast-food-gestorben-ld.104548 (21.10.2017).

17 Fischer, Joschka: „Er hat mein Land besser gemacht“. Siebeck praktizierte und lehrte Westbindung. http://www.zeit.de/2016/30/nachruf-wolfram-siebeck-joschka-fischer (12.11.2017).

18 Strobel y Serra, Jakob: Esst euch glücklich! http://www.faz.net/aktuell/feuilleton/medien/ zum-tod-des-kritikers-wolfram-siebeck-14331492.html (8.7.2016).

19 Vgl. https://www.tagesschau.de/multimedia/sendung/ts-14893.html, 00:15:51-00:16:17 (28.10.2017).

20 Zum Diskurs über kulinarische Ästhetik gehören nicht die Publikationen von Ernährungsberatern und Gesundheitsratgebern. Vgl. dazu Siebeck, Wolfram: Das Haar in der Suppe hab' ich nicht bestellt. Erinnerungen eines Berufsessers. Frankfurt am Main: Eichborn 1992, S. 123: „Wo es unkulinarisch zugeht, lauert Gesundheit.“

21 Vgl. hierzu Hegner, Henrike: Esskultur und Lebensstil. Medienanalyse ausgewählter Kochsendungen im Fernsehen. Marburg: Tectum 2015.

22 Eine der besten Werbungen für regionale Nahrungs- und Genussmittel bietet das „Kuratorium Kulinarisches Erbe Österreich“ an: https://www.kulinarisches-erbe.at (13.10.2017). 
neuer Medien, etwa Jürgen Dollases Esspapier. Kochbuchkritiken, ${ }^{23}$ Lutz Geißlers Plötzblog. Selbst gutes Brot backen ${ }^{24}$ oder der immer noch online abrufbare Blog Wo isst Wolfram Siebeck. ${ }^{25}$ Unübersehbar ist die Flut an Publikationen von Sachbüchern, Fachzeitschriften und Magazinen, die sich mit Genuss und Zubereitung von Speisen und Getränken befassen. In der frühen Bundesrepublik waren Arne Krüger und Walter Bickel ${ }^{26}$ prominent als Autoren, die sich mit Kulinarischem befassten. Seit den 1970er Jahren nahmen die überregionalen Zeitungen Gastronomiekritiken in ihre Feuilletons auf. Die nun breiter rezipierte Debatte bestimmten Klaus Besser, ${ }^{27}$ Gert von Paczensky ${ }^{28}$ und vor allem Wolfram Siebeck. ${ }^{29}$ Die genannten Autoren orientierten sich an der französischen Haute Cuisine und plädierten dafür, deren Niveau in der Bundesrepublik Deutschland zu etablieren. In der DDR galt Ursula Winnington als Protagonistin für gutes Essen. ${ }^{30}$ Ihre Vorliebe für die internationale und besonders die asiatische Küche verlangte unter den Rahmenbedingungen der Planwirtschaft allerdings schon Kompromisse bei den Zutaten. Rückblickend konstatiert sie: „In der DDR gab es wenig Extras, dafür aber alle Grundnahrungsmittel. Es lag an der Fantasie des Kochs, was Leckeres zu zaubern.“31

Die kulinarischen Entwicklungen von Bundesrepublik und DDR haben viele Parallelen: von der Rationierung in der Nachkriegszeit über den Massenkonsum vor allem von Fleisch bis zur Internationalisierung der Küche. Dennoch sind die Unterschiede unübersehbar. Während die Bundesrepublik freien Zugang zum Weltmarkt hatte, existierten in der DDR für alle Produkte, die mit harten Devisen gekauft werden mussten, Engpässe. Mit der Produktion von planwirt-

$23 \mathrm{http}: / /$ www.faz.net/aktuell/feuilleton/buecher/esspapier/ (13.10.2017).

24 https://www.ploetzblog.de (13.10.2017).

25 Vgl. etwa Siebeck, Wolfram: $36: 1$ = Noch mehr Kochbücher. http://wo-isst-siebeck.de/ 2011/11/36-1-noch-mehr-kochbucher (13.10.2017).

26 Abgesehen von Wikipedia-Artikeln existieren weder über Krüger noch über Bickel Biografien.

27 Besser gründete 1978 die Zeitschrift Besser's Gourmet Journal, die nach mehreren Namenänderungen heute noch als Der Feinschmecker erscheint. Abgesehen von einem WikipediaArtikel existiert noch keine Biografie über Besser.

28 Vgl. Paczensky, Gert von: Journalist mit Appetit. Köln: Walter Hädecke 2003.

29 Vgl. Siebeck, Haar in der Suppe (wie Anm. 20). Eine umfangreichere, noch nicht edierte Autobiografie Siebecks befindet sich seit Kurzem im Bestand der SLUB Dresden.

30 Abgesehen von einem Wikipedia-Artikel existiert noch keine Biografie über Winnington.

31 Hix, Jeanette: „Kerbel hilft dem Opa auf die Bärbel“. Ursula Winnington. Damals wie heute gibt sie heiße Liebestipps. Liebesgewürze. http://www.berliner-kurier.de/-kerbel-hilft-demopa-auf-die-baerbel-ursula-winnington-damals-wie-heute-gibt-sie-heisse-liebestipps-liebesgewuerze-21537616 (18.9.2017). 
schaftlicher Versorgung ${ }^{32}$ kontrastierte eine immer ausdifferenziertere marktwirtschaftliche Spezialisierung des Angebots in Westdeutschland. International renommierte Spitzenköche wie Eckhart Witzigmann oder auch eine öffentlich etablierte Gastronomiekritik fehlten in der DDR. Der Rückblick auf die Küche der DDR bildet seit 1990 einen eigenen Diskurs, der vorwiegend von Sachbuchautoren geführt wird. ${ }^{33}$ Die Debatte über die Gourmetküche in den neuen Bundesländern entfachte Wolfram Siebeck mit einer Karte über seine „Futterplätze“, die davon abriet, mit Ausnahme Berlins in Ostdeutschland Restaurants aufzusuchen. ${ }^{34}$ Siebeck selbst hat 2013 seine Meinung nach einer Verkostungsreise durch Sachsen revidiert und von „knospenden Landschaften“ gesprochen. ${ }^{35}$ Inzwischen haben auch andere renommierte Gastronomiekritiker die Kochkunst der neuen Bundesländer gewürdigt. ${ }^{36}$

Seit der Jahrtausendwende veränderte in der Bundesrepublik Jürgen Dollase den bis dahin feuilletonistischen Stil der Gastronomiekritik $\mathrm{zu}$ einem reflektierten Genre, indem er durch Parallelisierungen und Transfers von Begriffen aus der künstlerischen und musikalischen Ästhetik eine kulinarische Analy-

32 Zur industriellen Produktion von Nahrungsmitteln in der DDR vgl. Poutrus, Patrice G.: Die Erfindung des Goldbroilers. Herrschaftssicherung durch Konsumentwicklung in der DDR. Köln, Weimar, Wien: Böhlau 2002.

33 Vgl. etwa Voigt, Jutta: Der Geschmack des Ostens. Vom Essen, Trinken und Leben in der DDR. Berlin: Gustav Kiepenheuer 2005; Scheffler, Ute (Hrsg.): Alles Soljanka oder wie? Das ultimative DDR-Kochbuch 1949-1989. Leipzig: Bechtermünz 2000; Hoebel, Wolfgang: Vom Pagen im Hotel Adlon bis zum Mauerfall. Norderstedt: Books on Demand [2016]. Zu den wenigen wissenschaftlichen Studien gehören: Kochan, Thomas: Blauer Würger. So trank die DDR. Berlin: Aufbau 2011; Gries, Rainer: Produkte als Medien. Kulturgeschichte der Produktkommunikation in der Bundesrepublik und der DDR. Leipzig: Leipziger Universitätsverlag 2003; Huijzer, Marlise: Der moderne Ossi und sein Ostprodukt. Die Bedeutung ehemaliger DDR-Produkte für Jugendliche in Ostdeutschland. Marburg: Tectum 2008.

34 Vgl. Siebeck, Wolfram: Meine Futterplätze. In: ZEITmagazin vom 25.9.2008, S. 10-11.

35 Vgl. http://wo-isst-siebeck.de/2013/11/knospende-landschaften-1/ (21.10.2017).

36 Vgl. etwa Strobel y Serra, Jakob: Schönen Gruß vom starken August. In: Frankfurter Allgemeine Zeitung vom 19. Mai 2017, S. 12: „Stefan Hermann hat sich mit einem gastronomischen Kleinkönigreich ,bean \& beluga“ wie kaum ein Zweiter um den kulinarischen Aufbau Ost in Dresden verdient gemacht. Doch die Arbeit ist noch lange nicht vollbracht." Strobel y Serra, Jakob: Das Gänsestopflebertäuschungsmanöver. In: Frankfurter Allgemeine Zeitung vom 7. Juli 2017, S. 12: „Benjamin Biedlingmaier lässt sich in seinem Restaurant ,Caroussel` von der barocken Opulenz Dresdens nicht beeindrucken und treibt lieber Schabernack im Dienste des guten Geschmacks.“ Strobel y Serra, Jakob: Der große Bruder des kleinen Sterntalermädchens. In: Frankfurter Allgemeine Zeitung vom 1. September 2017, S. 14: „Stephan Mießner wollte in seinem Restaurant ,Elements‘ einfach nur so vor sich hin kochen. Das hätte ihm so passen können! Inzwischen zählt er zu den Fixpunkten der Dresdner Spitzengastronomie.“ 
tik entwickelte. ${ }^{37}$ Christoph Ribbat konstatierte, dass dies in einem größeren Kontext stand, denn die hedonistischen Restaurantkritiker des ausgehenden 20. Jahrhunderts seien inzwischen weithin von Food-Publizisten abgelöst worden, die nicht allein die kulinarische Kunst genießen, sondern ebenso die ethischpolitische Verantwortung ,im Kampf gegen Monokulturen oder gegen die Ausbeutung von Ressourcen und Menschen durch Nahrungsmittelkonzerne“ anmahnen. Zudem sei als Konsument der Kochkunst nicht mehr der Gourmet typisch, der Raffinesse und Eleganz eingefordert habe, sondern der Foody, dem es um Ursprünglichkeit und Nachhaltigkeit gehe. ${ }^{38}$

Der gesellschaftliche Diskurs zur Ernährung reicht aber über Feinschmeckerkreise und gesamtgesellschaftliche Debatten hinaus. Aus naturwissenschaftlicher Perspektive eröffnen Zugänge zur Kulinarik unter anderem Hervé This-Benckhard, ${ }^{39}$ Klaus Roth ${ }^{40}$ und Guy Crosby, ${ }^{41}$ vor allem aber Thomas A. Vilgis, der auch grundlegende Ansätze zur kulinarischen Konstruktion, etwa der Kombination von Aromen durch Food-Pairing (Überlappen von Aromen) und Food-Completing (Ergänzen einer Lücke im Aromenprofil der Hauptzutat), anbietet. $^{42}$

In der Kunst hat Daniel Spoerri seit Anfang der 1960er Jahre Essen als elementaren Bestandteil des kulturellen Lebens zum Inhalt seiner Arbeit gemacht. Bei Joseph Beuys finden sich die soziale Plastik des Mannes, der in der häuslichen Küche kocht, sowie das Konzept, den Anbau von Nahrungsmitteln als kreativen Widerstand gegen die Agrarindustrie zu verstehen. Peter Kubelkas künstlerische Arbeit des Speisenbaus versteht Essenszubereitung als älteste Kunstform, in der Farben und Formen, Temperaturen und Texturen komponiert

37 Grundlegend für Dollases Position vgl. Dollase, Jürgen: Geschmacksschule. Wiesbaden: Tre Torri 2005; Dollase, Jürgen: Kulinarische Intelligenz. Wiesbaden: Tre Torri 2006; Dollase, Jürgen: Kopf und Küche. Die Reise ins Innere des Geschmacks. Von der ersten Auster bis zu den besten Küchen Europas. Aarau, München: AT Verlag 2015.

38 Ribbat, Christoph: Im Restaurant. Eine Geschichte aus dem Bauch der Moderne. Berlin: Suhrkamp 2016, S. 181-183, das Zitat S. 182.

39 Vgl. This-Benckhard, Hervé: Rätsel und Geheimnisse der Kochkunst. Naturwissenschaftlich erklärt. München, Zürich: Piper 2001.

40 Vgl. Roth, Klaus: Chemische Delikatessen. Weinheim: Wiley-VCH 2007; Roth, Klaus: Chemische Leckerbissen. Weinheim: Wiley-VCH 2014.

41 Vgl. Crosby, Guy: Perfektion. Die Wissenschaft des guten Kochens. Bd. 1: Fleisch, Bd. 2: Gemüse u. Bd. 3: Backen. Berlin: Stiftung Warentest 2015 und 2016.

42 Vgl. u. a. Vierich, Thomas A. u. Thomas A. Vilgis: Aroma. Die Kunst des Würzens. Berlin: Stiftung Warentest 2012; Caviezel, Rolf u. Thomas A. Vilgis: Foodpairing. Harmonie und Kontrast. Lenzburg: FONA Verlag 2012; Caviezel, Rolf u. Thomas A. Vilgis: Das Parfüm der Küche. Der Schlüssel zum Genuss. Wiesbaden: Tre Torri 2014; Tzschirner, Hubertus u. Thomas A. Vilgis: Roh. Die neue Definition von Rohkost. Köln: Fackelträger Verlag 2014. 
werden. ${ }^{43}$ Der kunsthistorische Diskurs zum Essen akzeptierte inzwischen auch Beiträge aus der hoch entwickelten kulinarischen Ästhetik. Ferran Adrià, der Avantgardist der so genannten Molekularen Küche, nahm im Jahr 2007 an der Dokumenta 12 teil. ${ }^{44}$

Seit der Historiker Hans Jürgen Teuteberg und der Ethnologe Günter Wiegelmann mit ihrem gemeinsamen Buch über den Einfluss der Industrialisierung auf Ernährung die deutschsprachige Debatte der Geisteswissenschaft am Beginn der 1970er Jahre beflügelten, ${ }^{45}$ haben sich beispielsweise Germanisten wie Alois Wierlacher, Gerhard Neumann und Trude Ehlert, Romanisten wie Karin Becker und Christine Ott, Soziologen wie Eva Barlösius, Hans-Werner Prahl und Moritz Csáky, Ethnologen wie Konrad Köstlin, Gunther Hirschfelder, Thomas Hengartner, Bernhard Tschofen, Uwe Spiekermann und Barbara Krug-Richter, Historiker wie Ernst Schubert, Gerd Althoff, Roman Sandgruber, Hasso Spode, Jakob Tanner, Roman Rossfeld, Lothar Kolmer, Ingrid Haslinger und Hans Ottomeyer, Kulturwissenschaftler wie Peter Lesniczak und Maren Möhring sowie Philosophen, vor allem Harald Lemke, daran beteiligt. Ein Großteil der genannten Autoren befasste sich mit den Alltagsspeisen der meisten Menschen oder mit der Frage der sozialen Distinktion durch Nahrung, die Norbert Elias schon

43 Vgl. Lemke, Harald: Die Kunst des Essens. Eine Ästhetik des kulinarischen Geschmacks. Bielefeld: transcript 2007, S. 40, 62, 66 u. 85. Zur Eat Art in der Gegenwartskunst vgl. auch Kunsthalle Düsseldorf (Hrsg.): Eating the Universe. Vom Essen in der Kunst. Köln: DuMont 2009; Kunstforum International Bd. 159, April-Mai 2002: Essen und Trinken I u. Bd. 160, Juni-Juli 2002: Essen und Trinken II.

44 Vgl. Buergel, Roger u. Ruth Noak: Ein Künstler. In: Food for thought, Thought for food. Hrsg. von Richard Hamilton u. Vincente Todoli. Barcelona, New York: ACTAR 2009, S. 77; Arzak, Marta u. Jose Maria Pinot: Ferran Adriàs Beitrag zur documenta 12. In: Food for thought, S. 78-109; N. N.: Das Beispielmenü der documenta 12. In: Food for thought, S. 110-133; Giménez, Christina: Kulinarische Rückmeldung. In: Food for thought, S. 135-204; Emmerich, Alf: Der Kochkünstler.http://www.zeit.de/2007/01/Der_Kochkuenstler/komplettansicht (19.10.2017). 45 Vgl. Teuteberg, Hans Jürgen u. Günter Wiegelmann: Der Wandel der Nahrungsgewohnheiten unter dem Einfluss der Industrialisierung. Göttingen: Vandenhoeck \& Ruprecht 1972. Es existierte bereits vorher ein europäischer geisteswissenschaftlicher Diskurs zur Ästhetik des Essens, angestoßen durch Rumohr, Carl Friedrich von: Der Geist der Kochkunst. Stuttgart, Tübingen: Cotta'sche Buchhandlung 1822. Frühe deutschsprachige Standardwerke zur Historiografie der Ernährung sind Kudriaffsky, Eufemia von: Die historische Küche. Wien, Pest, Leipzig: Hartleben 1880; Lichtenfelt, Hans: Die Geschichte der Ernährung. Berlin: Georg Reimer 1913; Wiswe, Hans: Kulturgeschichte der Kochkunst. München: Moos 1970. Zum Diskurs in der europäischen Philosophie von der Antike bis in die Gegenwart vgl. Lemke, Harald: Ethik des Essens. Eine Einführung in die Gastrosophie. Berlin: Akademie Verlag 2007. 
1939 aufgeworfen hat. ${ }^{46}$ Das Spezifikum der europäischen Spitzenküche, „Kochen als Kunstform, als permanenten, kreativen Prozess, als unendliche, kulinarische Schöpfungsgeschichte“ ${ }^{47} \mathrm{zu}$ verstehen, wie es der Gastronomiekritiker und Reisejournalist Jakob Strobel y Serra formuliert hat, wird hierzulande im geisteswissenschaftlichen Diskurs nur selten thematisiert. Falls die europäische Charakteristik doch gelegentlich einmal zum Erkenntnisinteresse wird, unterliegt sie meist soziologisch vorgefertigten Deutungsmustern für den Geschichtsverlauf, statt als historiografische Erkenntnismöglichkeit für gesellschaftliche Wandlungsprozesse genutzt zu werden. Vor diesem Hintergrund fokussieren die Forschungen zur Ernährungsgeschichte am Institut für Geschichte der Technischen Universität Dresden die ästhetische Entwicklung der Kochkunst als europäisches Phänomen mit regionalen Bezügen. ${ }^{48}$ Einen zentralen Transmissionsriemen zwischen den Metropolen des Kontinents, in denen die exquisite Kochkunst blühte, bildete seit der Mitte des 17. Jahrhunderts die Diplomatie, die Spitzenküche als Werkzeug der Politik einsetzte. Ebenso lange präferierte die hohe Kochkunst Produkte im optimalen Reifezustand, der nur lokal bzw. regional erzielt werden kann. Die hohe Kochkunst und Tafelkultur gehören zu den kulturellen Techniken Europas, deren Publikationen nicht weniger Beachtung verdienen als die zu Kunst, Musik, Mode oder Architektur.

Ohne die Bestände der Sammlung, die Walter Putz im Jahr 2005 der SLUB übergeben hat und die Thomas Bürger als Bibliotheca Gastronomica in den Be-

46 Vgl. Elias, Norbert: Über den Prozeß der Zivilisation. Soziogenetische und psychogenetische Untersuchungen. Bd. 1: Wandlungen des Verhaltens in den weltlichen Oberschichten des Abendlandes. Basel: Haus zum Falken 1939, S. 139-145.

47 Strobel y Serra, Jakob: Europas Haute Cuisine. In: Frankfurter Allgemeine Zeitung vom 30. August 2017, S. 11: „Die schönste Tugend heißt Lasterhaftigkeit. Kochen als Kunst: Die Haute Cuisine ist die sinnvollste und sinnesfrohste Erfindung Europas und bis heute eine Kernkompetenz seiner Kultur, der kein anderer Kontinent Vergleichbares entgegenzusetzen hat.“

48 Vgl. Krüger, Benedikt: Gehobene und exquisite Küche in der Konsumgesellschaft. Dresden um 1900. Ostfildern: Thorbecke 2015; Kliewer, Mario: Geschmacksgaranten. Sächsische Hoflieferanten für exquisite Nahrungsmittel um 1900. Ostfildern: Thorbecke 2015; Iwanzeck, Marco: Dresden à la carte. Entstehung und kulinarische Einordnung der Restaurantkultur 1800 bis 1850. Ostfildern: Thorbecke 2016; Matzerath, Josef u. Annemarie Niering (Hrsg.): Tafelkultur Dresden um 1900. Ostfildern: Thorbecke 2013; Matzerath, Josef: Küche und Kochkunst des Dresdner Hofes um 1600. In: Deckardt, Johann: New Kunstreich und Nützliches Kochbuch [1611]. Hrsg. von Georg Jänecke u. Josef Matzerath. Ostfildern: Thorbecke 2014. S. 9-33; Iwanzeck, Marco u. Josef Matzerath: Franz Walcha und der Beginn der kulinarischen Moderne. In: Walcha, Franz: Der praktische Koch [1819]. Hrsg. von Josef Matzerath unter Mitarbeit von Marco Iwanzeck u. Angelika Rakowski. Ostfildern: Thorbecke 2014. S. 9-31; Matzerath, Josef: Küche und Kochkunst des Dresdner Hofes um 1900. In: Pötzsch, Ernst Max: Vollständige Herrschaftsküche des Kronprinzen von Sachsen. Hrsg. von Josef Matzerath unter Mitarbeit von Georg Jänecke, Mechthild Herzog u. Hannah Aehle. Ostfildern: Thorbecke 2013. S. 9-33. 
stand des Hauses eingegliedert hat, wären in Dresden Forschungen zur geschichtlichen Entwicklung der kulinarischen Ästhetik kaum möglich gewesen. Künftig wird die SLUB ebenso über den Nachlass von Wolfram Siebeck verfügen, der die Chance eröffnet, die Geschichte des Deutschen Küchenwunders zu rekonstruieren. Auch dieser Zugang zu einem bislang historiografisch unerschlossenen Thema verdankt sich unter anderem der Weitsicht und dem persönlichen Engagement Thomas Bürgers, der die Sammlung der SLUB um einen gesellschaftlich und kulturell fundamentalen Bereich erweiterte. Nahrungsaufnahme ist nicht nur eine existenzielle Voraussetzung für menschliches Leben, sondern Gourmandise auch eine Kunstform. Sie gehört gerade nach Dresden, wo der weltgewandte Europäer Carl Friedrich von Rumohr ( ${ }^{\star}$ 6. Januar 1785 in Reinhardtsgrimma, † 25. Juli 1843 in Dresden), der 1822 als Erster - nämlich vier Jahre, bevor Jean Anthelme Brillat-Savarin 1826 seine Schrift La Physiologie du Goût veröffentlichte - eine Kochkunsttheorie aufstellte, ${ }^{49}$ auf dem Inneren Neustädter Friedhof ruht.

Im bundesweiten Vergleich erreichte die SLUB als Forschungsbibliothek für kulinarische Ästhetik allein schon durch die Bibliotheca Gastronomica eine Position, wie sie für ältere Kochbücher allenfalls noch die Universitätsbibliothek in Frankfurt am Main oder die Staatsbibliothek zu Berlin besitzen. Die Frankfurter Universitätsbibliothek ist vor allem deshalb so gut mit kulinarischer Literatur ausgestattet, weil sie durch eine Dauerleihgabe des Vereins zur Förderung der Tafelkultur e. V., auf den die Bestände des im Zweiten Weltkrieg untergegangenen Kochkunstmuseums übergegangen sind, mit zirka 12000 Bänden aus dem 16. bis 21. Jahrhundert sowie zirka 100 handschriftlichen Rezeptsammlungen ausgestattet wurde. ${ }^{50}$ Als besonderer Bestand innerhalb der Frankfurter Universitätsbibliothek sind diese Kulinaria bislang nicht ausgewiesen. In der Staatsbibliothek zu Berlin verzeichnet der Katalog viele alte Kochbücher leider als Kriegsverluste. Der SLUB ist zu wünschen, dass sie den von Thomas Bürger so erfolgreich begonnenen Sammelbereich kulinarischer Publikationen fortsetzen kann und zu dem zentralen Ort wird, der diesem Kulturthema im deutschsprachigen Kontext bislang fehlt.

49 Vgl. Lemke, Ethik (wie Anm. 45), S. 366.

50 Vgl. Deutsches Museum für Kochkunst und Tafelkultur, Frankfurt am Main: http://www. tafelkultur.com/verein-stiftung/sammlungen/ (19.10.2017). 